\title{
Pericardium: structure and function in health and disease
}

\author{
Maria Jaworska-Wilczynska ${ }^{1}$, Pawel Trzaskoma ${ }^{2}$, \\ Andrzej A. Szczepankiewicz ${ }^{2}$, Tomasz Hryniewiecki ${ }^{1}$ \\ ${ }^{1}$ Department of Acquired Cardiac Defects, Institute of Cardiology, Warsaw, Poland \\ ${ }^{2}$ Nencki Institute of Experimental Biology, Warsaw, Poland
}

\begin{abstract}
Normal pericardium consists of an outer sac called fibrous pericardium and an inner one called serous pericardium. The two layers of serous pericardium: visceral and parietal are separated by the pericardial cavity, which contains 20 to $60 \mathrm{~mL}$ of the plasma ultrafiltrate.

The pericardium acts as mechanical protection for the heart and big vessels, and a lubrication to reduce friction between the heart and the surrounding structures.

A very important role in all aspects of pericardial functions is played by mesothelial cells. The mesothelial cells form a monolayer lining the serosal cavity and play an important role in antigen presentation, inflammation and tissue repair, coagulation and fibrinolysis. The two major types of mesothelial cells, flat or cuboid, differ substantially in their ultrastructure and, probably, functions. The latter display abundant microvilli, RER, Golgi dense bodies, membrane-bound vesicles and intracellular vacuoles containing electron-dense material described as dense bodies. The normal structure and functions of the pericardium determine correct healing after its injury as a result of surgery or microbial infection. The unfavorable resolution of acute or chronic pericarditis leads to the formation of adhesions between pericardial leaflets which may lead to serious complications. (Folia Histochemica et Cytobiologica 2016, Vol. 54, No. 3, 121-125)
\end{abstract}

Key words: pericardium; mesothelial cells; ultrastructure; 3D; SBF-SEM; pericarditis; leaflets adhesions; tissue repair

\section{Introduction}

Pericardium (from the Greek " $\pi \varepsilon \rho \iota$, " — around and " $\kappa \alpha \rho \delta \iota o v, "$ - heart) is a sac surrounding the heart and big vessels protecting them against injury and infection, and fixing the heart to the mediastinum. It consists of an outer part called fibrous pericardium and an inner layer called serous pericardium. The serous pericardium includes a visceral layer (epicardium) covering the heart and the roots of the big vessels, and the parietal layer overlying the fibrous pericardium. Both layers are separated by a slit-like pericardial cavity, which contains 20 to $60 \mathrm{~mL}$ of the plasma ultrafiltrate [1].

Correspondence address: M. Jaworska-Wilczynska, M.D., Ph.D. Department of Acquired Cardiac Defects Institute of Cardiology, Warsaw, Poland e-mail: mjawil@gmail.com

\section{The ultrastructure of the pericardium}

According to the textbook view on the serous pericardium, it consists of a single layer of flat epithelial cells of mesodermal origin, i.e. the mesothelium, resting on basal lamina underlined by a submesothelial layer of connective tissue [2]. However, several detailed ultrastructural studies on both, visceral and parietal, pericardial leaflets have indicated that the reality is more complex [3-7]. The most striking element of this complexity is the presence of (at least) two distinct mesothelial cell forms, the flat one and the cuboidal one. The flat cells have a thin cytoplasm raised over the centrally positioned nucleus. Their organellar content is scant, with few mitochondria, poorly developed endoplasmic reticulum and some intracellular vesicles. The Golgi apparatus is infrequently present in electron micrographs. The signs of some endo/ /exo-cytotic or trans-cytotic activity are visible as vesicle formation at both the apical and basal cell membranes. The apical membrane is covered by short and 

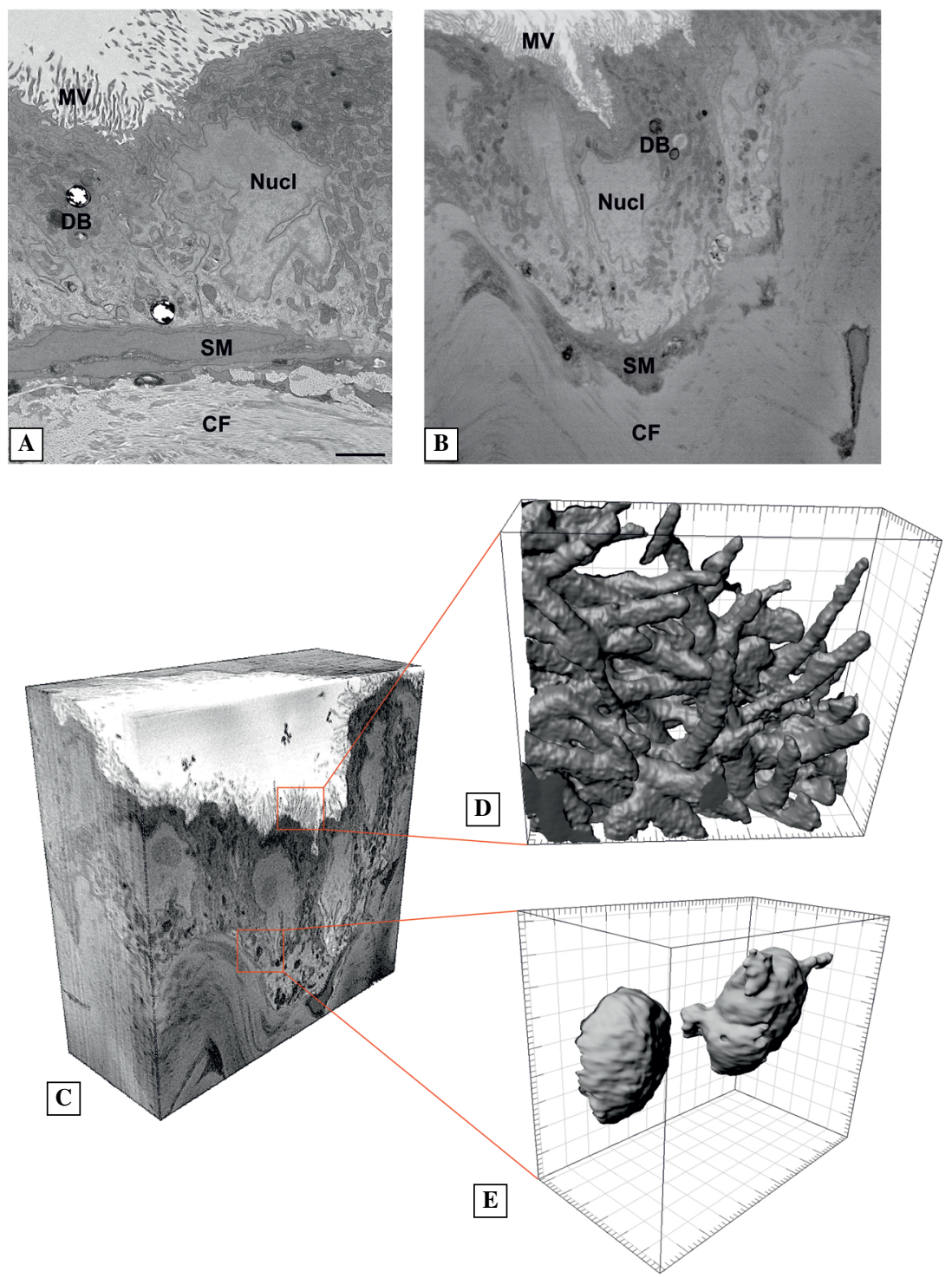

Figure 1A. A single transmission electron micrograph of the human parietal pericardium; B. A stack of 100 electron-microscopic images obtained using a serial block-face scanning electron microscopy (SBF-SEM); the stack spans $25 \times 25 \times 5 \mu$ m; C. A three-dimensional (3D) view of the stack shown in B; D. Enlarged 3D rendering of the surface microvilli;

E. Enlarged 3D reconstructions of the dense bodies. Abbreviations: MV — microvilli; DB — dense body; Nucl — nucleus; $\mathrm{SM}$ - submesothelial layer; CF — collagen fibers. The scale bar, $1 \mu \mathrm{m}$, refers to A and B.

scant microvilli [3-7]. In contrast, the cuboidal cells are rich in organelles, with numerous mitochondria, very well elaborated rough endoplasmic reticulum and abundant Golgi stacks [3-7] (Figure 1). There are numerous vesicles, vacuoles and multivesicular bodies, as well as the signs of the robust vesicle formation at the cell membranes in the form of either coated or uncoated vesicles being in the process of internalization from or budding of the cell membranes (Figure 1). In addition, the cytoplasm of cuboidal cells contains numerous vesicles/vacuoles filled entirely or partially with an electron-dense material, sometimes displaying a multilamellar appearance, often described as dense bodies [8] (Figure 1). These structures are believed to contain surfactant-like substances, similar to those produced by type II pneumocytes [8]. The apical surface of cuboidal cells is covered by abundant microvilli, having the length of up to $3 \mu \mathrm{m}$ [3-7]; some of the microvilli bifurcate near their ends. Both cell types occasional bear primary cilia, and are covered by a thick layer of glycocalyx [3-5].

Junctional complexes between adjacent mesothelial cells (both flat and cuboidal) consist of desmosomes, which reinforce intercellular adhesion and 
tight junctions, which form permeability barriers [3-7]. Accordingly, the pericardial layers are impermeable to the particles of the size ranging from 10 to $30 \mathrm{~nm}$; however, they do allow for passing the particles that are smaller than $6 \mathrm{~nm}$, such as horseradish peroxidase molecules [9]. Moreover, several authors also describe the presence of large irregular intercellular spaces that appear to arise through the coalescence of the electron-lucent vesicles, and are "sealed" up and down by the tight junctions [3-7]. It remains to be determined, whether such intercellular spaces evident at 2D micrographs represent in fact some kind of tortuous channels linking directly the pericardial cavity with the submesothelial spaces, allowing to pass even large particles or cells. The large direct channels called the "stomata" have been described in other serosal membranes, i.e. pleura and peritoneum $[5,6]$. Interestingly, in rodents, Nakatani et al. [10] described the presence of fenestrations lined with mesothelial cells, up to $50 \mu \mathrm{m}$ in diameter, that directly connected the parietal pericardium with the pleural cavity [10].

Traditionally, the cuboidal mesothelial cells have been considered to be a predominant mesothelial cell type in the visceral pericardium, whereas flat mesothelial cells are thought to predominate in the parietal pericardium $[3,5,6]$. At the parietal side, the cuboidal cells have been described to appear solitary or in small groups [5, 6]. However, the findings by Ishihara et al. (1980), and our own recent observations by $3 \mathrm{D}$ electron microscopy (Figure 1) show that the vast majority of the parietal mesothelial cells have, in fact, the cuboidal appearance. It is also possible that the flat and cuboidal cells represent distinct states of cell differentiation/activation. Some investigators described the existence of cells which have ultrastructural features that are intermediate between the two aforementioned phenotypes [5, 6]. It has also been shown that the number of the cuboidal cells in serosal membranes (including pleura and peritoneum) increases under various pathological conditions ([6] and further).

The submesothelial layer differs strikingly between the visceral and the parietal mesothelium [7, 11]. In the epicardium it consists of a loose connective tissue containing some collagen and elastic fibers. It is rich in cells such as fibroblasts, undifferentiated mesenchymal cells, telocytes, as well as adipocytes (predominantly along coronary vessels), blood and lymphatic vessels, and nerve fibers [11]. This tissue merges with the underlying myocardium. In contrast, in the parietal pericardium, the submesothelial layer is thin, contains few fibrocyte-like cells, some elastic fibers, and an abundant wavy collagen fibers [7] [Jaworska-Wilczynska et al., in preparation]. At some places in the parietal, but not in the visceral pericardium, the mesothelial covering becomes discontinuous, and the submesothelial layer becomes exposed to the pericardial cavity. At such sites the submesothelial layer transforms into the milky spots-like structure, containing very abundant macrophages and lymphocytes, as well as lymphatic capillaries [12]. The exact function of these structures is unknown; however, by providing a direct access of the pericardial fluid to the submesothelial lymphatic system, they allow the effective drainage of the pericardial space [13]. They have been also suggested to have immune-related functions [12]. The submesothelial layer merges with an underlying fibrosa containing thick collagen fiber bundles with alternating orientation. The fibrosa transforms gradually into the dense connective tissue of the fibrous pericardium [7].

The mesothelium is a slowly renewing tissue with $0.16-0.5 \%$ of cellsundergoingmitosisat any one time[14]. The primary function of the mesothelium is to provide a slippery surface, but it is also involved in the transport of solutes and cells, immune defenses and tissue regeneration [14].

\section{Acute inflammation and tissue repair}

Cardiac surgical procedures result in the physical damage and an influx of blood into the pericardial cavity. Using both, light- and electron-microscopy Nkere et al. (1994) [15] described the dynamics of degenerative changes affecting mesothelium and serosa during the course of coronary artery bypass operation. In the biopsies taken at different time points after the pericardiotomy, the authors found progressive alterations of the pericardium, beginning with the transformation of the mesothelial cells from flat to cuboidal shape, and the infiltration of the submesothelial space by leukocytes (75 min after pericardiotomy), followed by death and desquamation of mesothelial cells, exposure of the underlying connective tissue, intense transmigration of leukocytes onto the surface of the pericardium, and the deposition of fibrin (135 $\mathrm{min}$ after the pericardiotomy) [15]. Further stages of serosal damage and, eventually, healing, can be inferred from animal studies on experimental hemothorax [16] or pneumonectomy [17]. Usually the healing process completes after 5-8 days, as a result of proliferation of mesothelial cells and their epithelial-to-mesenchymal transition (EMT), allowing transmigration and settlement at the denuded areas [18-19]. The pericardial regeneration is believed to be triggered by the loss of contact inhibition, and by soluble mediators released from activated cells [18]. The exposed basal lamina could be also repopulated by 
cells free-floating in the pericardial fluid [19]. The mesothelial repair is different from that occurring in other epithelia, as regrowth appears diffusely across the injured surface [14]. After the return to primary intact constitution, the cells undergo transformation to mesothelial-like phenotype $[18,19]$.

\section{Chronic inflammation and intrapericardial adhesions}

In case of an unfavorable outcome, i.e. regeneration of mesothelium, extensive adhesions between pericardial leaflets are formed at the sites of fibrin deposition which become populated by connective tissue cells and invaded by capillaries. The adhesions are seemed to be unavoidable consequence of the surgery and may complicate rethoracotomy due to injury of the heart and big vessels and uncontrolled bleeding [20].

Proposed events leading to chronic inflammation of serosal membranes including peritoneum, are extrinsic factors such as infections, surgical manipulation of the pericardium, bleeding to the pericardial space, extracorporeal circulation, cardiac failure and uremia, and intrinsic factors such as the loss of mesothelial cells and exposure of the submesothelial layer, EMT, abundance of macrophages, and decreased fibrinolytic activity of mesothelial cells, observed also in peritoneum and pleura $[14,20,21]$.

The most important cause of the formation of pericardial leaflets' adhesions is the denudation of the submesothelial layers, since fibrin, platelets and inflammatory cells adhere to these surfaces. Fibrin deposition is formed early during the cardiotomy [15] and this is a consequence of the inadequate fibrinolytic activity of mesothelial cells [20]. Otherwise during the injury, the mesothelial cells participate in inflammation. They secrete various proinfammatory and immunomodulatory mediators, including products of the coagulation cascade, chemokines, cytokines, growth factors, prostaglandins, reactive oxygen and nitrogen species, and proteolytic enzymes [18]. They present also potent procoagulant activity due to the secretion of tissue factor, the main cellular activator of the extrinsic coagulation cascade [22]. Secretion of chemokines by mesothelial cells promotes trans-mesothelial migration of neutrophils and monocytes/ /macrophages into the serosal space [18, 23]. This is facilitated by the expression of integrins and adhesion molecules on mesothelium (ICAM-1) and leukocytes (LFA-1, Mac-1) [14, 24]. However, mesothelial cells also participate in the resolution of inflammation by specific integrin-mediated regulation involving very late antigens (VLA-4 and VLA-5) expression [14]. A very important process which occurs during the pericardial/pleural/peritoneal injury is the EMT. During this process the mesothelial cells undergo a progressive loss of the epithelial phenotype and develop fibroblast-like characteristics which allows them to contribute to the development of fibrosis and angiogenesis in the submesothelial zone [25]. Phenotypically, the mesothelial cells loose their cobblestone phenotype and cytokeratin content [25]. The main mediator of the transformed mesothelial cell is TGF- $\beta$, which promotes profibrotic processes including fibroblast activation, collagen deposition, inhibition of fibrinolysis, and neoangiogenesis. TGF- $\beta$ induces $\beta$-catenin expression which, together with AP-1 transcription factor, activates matrix metallopeptidase 9 (MMP-9) synthesis and extracellular matrix invasion by transformed mesothelial cells [25]. As the consequence of the inflammation, the connective tissue bands are formed on the pericardial surfaces covering fibrin accumulation [20]. In parallel to fibrosis angiogenesis and vessels formation occurs in adhesions [20]. One month after surgery there is persistent connective tissue formation that fills up the pericardial space and results in the adherence of the pericardial leaflets [20].

\section{Pericardial fluid drainage}

The pericardial cavity contains $20-60 \mathrm{~mL}$ of plasma ultrafiltrate $[13,26]$. Its cellular components include some mesothelial cells, lymphocytes $(53 \%)$, neutrophils (31\%), macrophages (12\%), eosinophils (1.7\%) and basophils $(1.2 \%)[13,27]$. The osmolality of pericardial fluid is lower than plasma. The concentrations of $\mathrm{Na}^{+}, \mathrm{Cl}^{-}, \mathrm{Ca}^{2+}$ and $\mathrm{Mg}^{2+}$ are lower and $\mathrm{K}^{+}$level is higher than in plasma [13]. There is a strong evidence that the pericardial fluid derives mainly as a transudate of epicardial capillaries and probably also the parietal ones [28]. The parietal pericardium lymphatic capillaries are responsible for the fluid drainage [13]. In pathological conditions during infection or after cardiotomy the physiological balance between the production and drainage of the fluid is disturbed, leading to pericardial effusion The inflammation leading to congestion and widening of the capillaries, and deposition of fibrin and connective tissue on the parietal layer of pericardium, impairing lymphatic drainage, are the reason of this imbalance [20].

\section{Conclusions}

The studies of pericardium structure and function have shown an important role of the mesothelial cells. They participate in the modulation of inflammatory processes, tissue repair, coagulation, fibrinolysis and 
are also involved in the transport of solutes. Impaired healing of the pericardium leads to the formation of intrapericardial adhesions that may impair heart's function.

\section{Acknowledgements}

The authors thank Dr. Grzegorz M. Wilczynski for fruitful discussions. The work was supported by the Statutory Funds of the Institute of Cardiology.

\section{References}

1. Khandaker MH, Espinosa RE, Nishimura RA et al. Pericardial disease: diagnosis and management. Mayo Clin Proc. 2010;85:572-593. doi: 10.4065/mcp.2010.0046.

2. Krstic RV. Human microscopic anatomy. $3^{\text {rd }}$ ed. Berlin: Springer-Verlag; 1991.

3. Kluge T, Hovig T. The ultrastructure of human and rat pericardium. I. Parietal and viscera mesothelium. Acta Pathol Microbiol Scand. 1967;71:529-546. PMID: 4869325.

4. Kluge T, Hovig T. The ultrastructure of human and rat pericardium. II. Intercellular spaces and junctions. Acta Pathol Microbiol Scand. 1967;71:547-563. PMID: 4869326.

5. Michailova KN. The serous membranes in the cat. Electron microscopic observations. Ann Anat. 1996;178:413-424. PMID: 8931852.

6. Michailova KN, Usunoff KG. Serosal membranes (pleura, pericardium, peritoneum). Normal structure, development and experimental pathology. Adv Anat Embryol Cell Biol. 2006;183:1-144. PMID: 16570866.

7. Ishihara T, Ferrans VJ, Jones M et al. Histologic and ultrastructural features of normal human parietal pericardium. Am J Cardiol. 1980;46:744-753. PMID: 7435384.

8. Michailova KN. Mesothelial lamellar bodies in norm and experimental conditions. Transmission and scanning electron microscopic observations on the peritoneum, pleura and pericardium. Anat Embryol (Berl). 2004;208:301-309. doi: 10.1007/s00429-004-0390-5.

9. Kluge T. The permeability of mesothelium to horseradish peroxidase. A light and electron microscopic study, with special reference to the morphology of pericardial mast cells. Acta Pathol Microbiol Scand. 1969;75:257-269. PMID: 5822964.

10. Nakatani, T, Shinohara H, Fukuo Y et al. Pericardium of rodents: pores connect the pericardial and pleural cavities. Anat Rec. 1988;220:132-137. PMID: 3354856.

11. Gherghiceanu M, Popescu LM. Human epicardium: ultrastructural ancestry of mesothelium and mesenchymal cells. J Cell Mol Med. 2009;13:2949-2951. doi: 10.1111/j.15824934.2009.00869.x.

12. Takada K, Otsuki Y, Magari S. Lymphatics and pre-lymphatics of the rabbit pericardium and epicardium with special emphasis on particulate absorption and milky spot-like structures. Lymphology. 1991;24:116-124. PMID: 1753803.

13. Vogiatzidis K, Zarogiannis SG, Aidonidis I et al. Physiology of pericardial fluid production and drainage. Front Physiol. 2015;18:6-62. doi: 10.3389/fphys.2015.00062.

14. Mutsaers SE, Birnie K, Lansley S et al. Mesothelial cells in tissue repair and fibrosis. Front Pharmacol. 2015;9:6-113. doi: 10.3389/fphar.2015.00113.

15. Nkere UU, Whawell SA, Sarraf CE et al. Perioperative histologic and ultrastructural changes in the pericardium and adhesions. Ann Thorac Surg. 1994;58:437-444. PMID: 8067846.

16. Michailova KN. Electron microscopic alterations of the rat's pleura after experimental haemothorax. Ann Anat. 2004;186:115-125. doi: 10.1016/S0940-9602(04)80022-0.

17. Michailova KN. Electron microscopic observations on the visceral and parietal rat's pleura after contralateral pneumonectomy. Eur J Morphol. 2001;39:47-56. PMID: 11262630.

18. Mutsaers SE. Mesothelial cells: their structure, function and role in serosal repair. Respirology. 2002;7:171-191. PMID: 12153683.

19. Foley-Comer AJ, Foley-Comer AJ, Herrick SE et al. Evidence for incorporation of free-floating mesothelial cells as a mechanism of serosal healing. J Cell Sci. 2002;115:1383-1389. PMID: 11896186.

20. Cannata A, Cannata A, Petrella D et al. Postsurgical intrapericardial adhesions: mechanisms of formation and prevention. Ann Thorac Surg. 2013;95:1818-1826. doi: 10.1016/j. athoracsur.2012.11.020.

21. Lai KN, Leung JC. Inflammation in peritoneal dialysis. Nephron Clin Pract. 2010;116:c11-c18. doi: 10.1159/000314544.

22. Hellebrekers BW, Kooistra T. Pathogenesis of postoperative adhesion formation. Br J Surg. 2011;98:1503-1516. doi: 10.1002/bjs.7657.

23. Wilshaw SP, Burke D, Fisher J. Investigation of the antiadhesive properties of human mesothelial cells cultured in vitro on implantable surgical materials. J Biomed Mater Res B Appl Biomater. 2009;88:49-60. doi: 10.1002/jbm.b.31148.

24. Bellingan GJ, Xu P, Cooksley H et al. Adhesion molecule-dependent mechanisms regulate the rate of macrophage clearance during the resolution of peritoneal inflammation. $J \operatorname{Exp}$ Med. 2002;196:1515-1521. PMID: 12461086.

25. Germani A, Foglio E, Capogrossi MC et al. Generation of cardiac progenitor cells through epicardial to mesenchymal transition. J Mol Med (Berl). 2015;93:735-748. doi: 10.1007/ s00109-015-1290-2.

26. Vesely TM, Cahill DR. Cross-sectional anatomy of the pericardial sinuses, recesses, and adjacent structures. Surg Radiol Anat. 1986;8:221-227. PMID: 3107145.

27. Kopcinovic LM, Culej J. Pleural, peritoneal and pericardial effusions - a biochemical approach. Biochem Med (Zagreb). 2014;24:123-237. doi: 10.11613/BM.2014.014.

28. Stewart RH, Rohn DA, Allen SJ et al. Basic determinants of epicardial transudation. Am J Physiol. 1997;273(3 Pt 2): H1408-H1414. PMID: 9321832.

Submitted: 11 June, 2016

Accepted after reviews: 27 July, 2016 Available as AoP: 16 August, 2016 